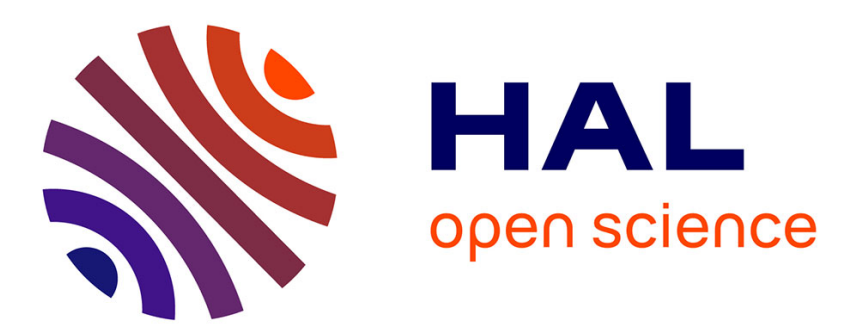

\title{
A two-dimensional computational study of gas flow regimes past of square cylinder confined in a long microchannel
}

Kiril S Shterev, Stefan K Stefanov

\section{- To cite this version:}

Kiril S Shterev, Stefan K Stefanov. A two-dimensional computational study of gas flow regimes past of square cylinder confined in a long microchannel. European Journal of Mechanics - B/Fluids, 2017. hal-02370462

\section{HAL Id: hal-02370462 \\ https://hal.science/hal-02370462}

Submitted on 19 Nov 2019

HAL is a multi-disciplinary open access archive for the deposit and dissemination of scientific research documents, whether they are published or not. The documents may come from teaching and research institutions in France or abroad, or from public or private research centers.
L'archive ouverte pluridisciplinaire HAL, est destinée au dépôt et à la diffusion de documents scientifiques de niveau recherche, publiés ou non, émanant des établissements d'enseignement et de recherche français ou étrangers, des laboratoires publics ou privés. 


\title{
A two-dimensional computational study of gas flow regimes past of square cylinder confined in a long microchannel
}

\author{
Kiril S. Shterev*, Stefan K. Stefanov \\ Institute of Mechanics, Bulgarian Academy of Sciences, \\ Acad. G. Bonchev Str., Block 4, Sofia 1113, Bulgaria
}

\begin{abstract}
In this paper, the transition between steady and unsteady regimes of the two-dimensional gas flow past a moving square cylinder confined between two parallel plates with equal temperatures was investigated for different subsonic and transonic speeds and blockage ratios $\mathrm{B}=3,5,10$ and 20 ( $\mathrm{B}$ is the ratio between microchannel height and square size). The influence of $\mathrm{B}$ on the drag coefficient of the square, pressure at stagnation point and transition between steady and unsteady regime were studied. A set of transition curves were obtained for different blockage ratios. The results showed a similar shape of the neutral curves, which shifts to lower Knudsen and Mach numbers when B decrease. A continuum approach based on the Navier-Stokes-Fourier equations was basically applied to all flow regimes. Navier-Stokes-Fourier equations was calculated numerically by using pressure based finite volume algorithm SIMPLE-TS. For the initial transition velocity slip regime, the results were compared to data obtained by using the particle Direct Simulation Monte Carlo (DSMC) method.
\end{abstract}

Keywords: transition, steady flow regime, unsteady flow regime, microflows, continuum model, SIMPLE-TS, molecular model, DSMC, SBT PACS: 47.15.Fe, 47.27.nd, 47.45.-n, 87.10.Rt 2000 MSC: 76F06, 76P05, 76M12, 82B80

\footnotetext{
*Corresponding author

Email addresses: kshterev@imbm.bas.bg (Kiril S. Shterev ), stefanov@imbm.bas.bg (Stefan K. Stefanov)
} 


\section{Introduction}

Micromechanical devices are associated with rapidly emerging technologies, where new potential applications are continuously being developed, and fluid flow regime is important for their design [1], [2]. For example, a gaseous flow past a small particle confined in microchannel is a typical microfluidic configuration, which is considered here. The flow regime could different significantly under different conditions. Thus, in this paper a variety of established two-dimensional steady and unsteady regimes of flow past a cylinder with square cross-section confined in a microchannel is investigated as a function of gas rarefaction (Knudsen number), velocity of the moving obstacle (Mach number) and blockage ratio of the channel (ratio between microchannel height and square size). To simplify the problem, we consider the equivalent inverse problem, in which the obstacle is fixed in a local coordinate system and gas and channel walls are moving in the opposite direction with given velocity. Usually, a microchannel gas flow is modelled by using either continuum approach with slip boundary conditions or by using rarefied gas dynamics methods. The Knudsen number (Kn), defined as a ratio of the mean free path $\ell_{\infty}^{*}$ to the macroscopic length scale of a physical system $L^{*}$,

$$
\mathrm{Kn}=\frac{\ell_{\infty}^{*}}{L^{*}}
$$

is a nondimensional parameter that determines the degree of gas rarefaction, and respectively, the degree of validity of the continuum model.

For larger Knudsen numbers, where the non-equilibrium effects are significant, a molecular approach, based on kinetic theory models [3], [4], or the particle DSMC (Direct Simulation Monte Carlo) method [5] has to be applied. In the present paper, we use both Navier-Stokes-Fourier continuum and molecular (DSMC) models to calculate the flow in the early transition regimes. The Navier-Stokes-Fourier continuum model $\underline{6]}$ with included statedepended transport coefficients determined by the first approximation of the Chapman-Enskog theory is used at low Knudsen numbers.

The peculiarity of fluid flows in and around micro-electro-mechanicalsystems (MEMS) could be related in most cases to low speed effects. The low Mach number typical for such flows makes pressure based methods very suitable to be used here. The pressure based algorithm SIMPLE-TS $\underline{7]}$ applied in the current study covers the entire slip regime.

The emergency of instability and prediction of a transition between different flow regimes is an important task in the study of gaseous flows around 
bodies with arbitrary shape. The laminar-turbulent properties of the flow field have a significant influence on skin friction and flow separation and, respectively on the lift and drag characteristics. Different transition prediction models have been developed to simplify both steady and unsteady flow regime predictions, see [8], [9].

In particular, investigations of the final state of incompressible fluid past a square with non-slip boundary conditions are widely reported [10]. By contrast, there are a few investigations of the problem in slip and transition regimes. We refer to Cheng and Liao [11], who have analyzed a gaseous flow past a square in the transition regime ( moderate and high Knudsen numbers) for Mach number ranged from 0.85 to 8. Here we investigate the final state of compressible viscous flow with slip boundary conditions as a function of Knudsen and Mach numbers, and blockage ratio in the case of a two-dimensional flow past a square cylinder in a long microchannel. The investigated Knudsen numbers are in the range from 0.002 to 0.02 . The free stream Mach numbers $(\mathrm{M})$ are in the range from 0.1 to 0.6 , while the local Mach number varies from 0.1 to $\sim 1.5$. In order to be able to calculate all problems with a unified algorithm, the iterative pressure based finite volume method (FVM) with improved convergence with respect to time step (SIMPLE-TS) was used [7]. A GPU (Graphical Processor Unit) version of algorithm SIMPLE-TS [12] with Initial Guess Extrapolation (IGE) [13] was used to speed-up the calculations.

A number of simulations by using the DSMC method has been performed to validate the SIMPLE-TS for moderate Knudsen numbers. The same DSMC algorithm has been applied previously in series of papers, [6], [14], [15], for studying the flow instability of 2D and 3D Rayleigh-Bénard flow of a rarefied gas as a function of Knudsen and Froude numbers.

\section{Continuum model equations}

The time evolution of the continuum model is described on the basis of the Navier-Stokes-Fourier (NSF) equations [6] for a compressible viscous gas with transport coefficients determined by the first approximation of the Chapman-Enskog theory for low Knudsen numbers.

The NSF equation system consists of the equations of continuity, momentums, energy and state for perfect gas. We introduce the nondimensional 
variables

$$
u=\frac{u^{*}}{V_{t h}^{*}}, \quad v=\frac{v^{*}}{V_{t h}^{*}}, \quad p=\frac{p^{*}}{p_{\infty}^{*}}, \quad T=\frac{T^{*}}{T_{w}^{*}}, \quad t=\frac{L^{*}}{V_{t h}^{*}}, \quad x=\frac{x^{*}}{L^{*}}, \quad y=\frac{y^{*}}{L^{*}},
$$

where $u$ is nondimensional horizontal velocity, $v$ is nondimensional vertical velocity, $p$ is nondimensional pressure, $T$ is nondimensional temperature, $t$ is nondimensional time, $x$ and $y$ are nondimensional coordinates of a Cartesian coordinate system, an asterisk denotes dimensional variables, infinity represent free stream conditions, $L^{*}$ is the dimensional reference length equal to the square size $\left(L^{*}=a^{*}\right.$, see Figure 1$), V_{t h}^{*}=\sqrt{2 R T_{w}^{*}}$ is the most probable molecular speed, $R$ is the gas constant, $T_{w}^{*}$ is dimensional temperature of channel and square walls, see Figure 1.

In our considerations, a hard-sphere gas description with viscosity coefficient $\mu$ and the heat conduction coefficient $\lambda$ (first approximations are sufficient) reads:

$$
\begin{gathered}
\mu=\mu_{h} \sqrt{T}, \mu_{h}=(5 / 16) \rho_{\infty}^{*} l_{\infty}^{*} V_{t h}^{*} \sqrt{\pi} \\
\lambda=\lambda_{h} \sqrt{T}, \lambda_{h}=(15 / 32) c_{p} \rho_{\infty}^{*} l_{\infty}^{*} V_{t h}^{*} \sqrt{\pi}
\end{gathered}
$$

where: $\rho_{\infty}^{*}$ is dimensional free stream density.

Other used variables are: nondimensional density is $\rho$, Mach number of $V_{t h}^{*}$ is $\mathrm{Ma}=V_{t h}^{*} / \sqrt{\gamma R T_{w}^{*}}=\sqrt{6 / 5}$, the free stream Mach number is $M=u_{s q}^{*} / \sqrt{\gamma R T_{w}^{*}}$, dimensional square velocity is $u_{s q}^{*}$, the Prandtl number is $\operatorname{Pr}=2 / 3, C_{\mu}=(5 / 16) \sqrt{2 \pi / \gamma}$ and $\gamma=c_{p} / c_{v}=5 / 3\left(c_{p}\right.$ and $c_{v}$ are the heat capacities at constant pressure and constant volume respectively).

Then, the system of unsteady nondimensional continuum equations can be expressed in a conservative form as follows:

$$
\begin{gathered}
\frac{\partial \rho}{\partial t}+\frac{\partial(\rho u)}{\partial x}+\frac{\partial(\rho v)}{\partial y}=0 \\
\frac{D u}{D t}=-\frac{1}{\gamma \rho \mathrm{Ma}^{2}} \frac{\partial p}{\partial x}+C_{\mu} \frac{2 \mathrm{Kn}}{\rho \mathrm{Ma}} \frac{\partial}{\partial x}\left(\sqrt{T} \frac{\partial u}{\partial x}\right) \\
+C_{\mu} \frac{\mathrm{Kn}}{\rho \mathrm{Ma}}\left\{\frac{\partial}{\partial y}\left[\sqrt{T}\left(\frac{\partial u}{\partial y}+\frac{\partial v}{\partial x}\right)\right]-\frac{2}{3} \frac{\partial}{\partial x}\left[\sqrt{T}\left(\frac{\partial u}{\partial x}+\frac{\partial v}{\partial y}\right)\right]\right\} \\
\frac{D v}{D t}=-\frac{1}{\gamma \rho \mathrm{Ma}^{2}} \frac{\partial p}{\partial y}+C_{\mu} \frac{2 \mathrm{Kn}}{\rho \mathrm{Ma}} \frac{\partial}{\partial y}\left(\sqrt{T} \frac{\partial v}{\partial y}\right) \\
+C_{\mu} \frac{\mathrm{Kn}}{\rho \mathrm{Ma}}\left\{\frac{\partial}{\partial x}\left[\sqrt{T}\left(\frac{\partial v}{\partial x}+\frac{\partial u}{\partial y}\right)\right]-\frac{2}{3} \frac{\partial}{\partial y}\left[\Gamma\left(\frac{\partial u}{\partial x}+\frac{\partial v}{\partial y}\right)\right]\right\}
\end{gathered}
$$




$$
\begin{gathered}
\frac{D T}{D t}=C_{\mu} \frac{\mathrm{Kn}}{\rho \mathrm{MaPr}}\left[\frac{\partial}{\partial x}\left(\sqrt{T} \frac{\partial T}{\partial x}\right)+\frac{\partial}{\partial y}\left(\sqrt{T} \frac{\partial T}{\partial y}\right)\right] \\
+\frac{(\gamma-1) C_{\mu} \mathrm{KnMa}}{\rho \quad} \sqrt{T} \Phi+\frac{(\gamma-1)}{\gamma \rho} \frac{D p}{D t} \\
p=\rho T
\end{gathered}
$$

where the dissipation function is given by:

$$
\Phi=2\left[\left(\frac{\partial u}{\partial x}\right)^{2}+\left(\frac{\partial v}{\partial y}\right)^{2}\right]+\left(\frac{\partial v}{\partial x}+\frac{\partial u}{\partial y}\right)^{2}-\frac{2}{3}\left(\frac{\partial u}{\partial x}+\frac{\partial v}{\partial y}\right)^{2}
$$

\section{Flow geometry and boundary conditions}

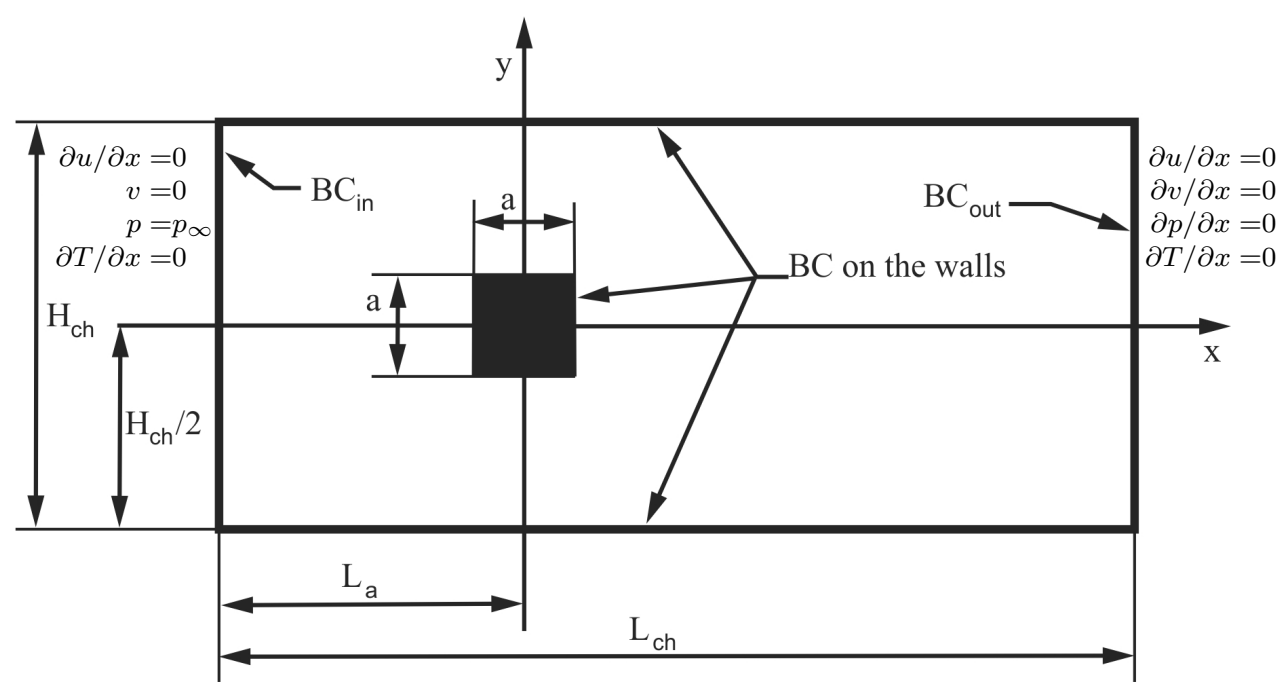

Figure 1: Flow geometry for a square cylinder with size $a$ confined in a channel with length $L_{c h}$, height $H_{c h}$ and boundary conditions $(\mathrm{BC})$ at inlet $\left(\mathrm{BC}_{\mathrm{in}}\right)$ and outlet $\left(\mathrm{BC}_{\mathrm{out}}\right)$.

The final state of the flow is determined for the case of a square cylinder with size $a$ confined in a plane microchannel (height $H_{c h}$ ) as shown in Figure 1. The considered blockage ratios $\left(B=H_{c h} / a\right)$ are $B=3,5,10$ and 20 . The distance from square to the channel inlet flow is $L_{a}$ (inlet length).

The considered equation system represents two equivalent problem descriptions with respect to the coordinate system (depending on the observer 
viewpoint). In the first case, the Cartesian coordinate system $(\overline{0} \overline{x y})$ is fixed to the stationary channel. The square cylinder is moving with constant velocity $-u_{s q}$ along $\bar{x}$-axes and the channel wall velocity is zero. The variables related to the coordinate system $\overline{0} \overline{x y}$ are denoted with overline. In the second case, a local Cartesian coordinate system $(0 x y)$ is fixed to the moving square cylinder. In the local coordinate system the velocity of the square cylinder is zero while the channel walls are moving with constant velocity $u_{s q}$. In order to use a computational grid fixed to the moving square cylinder, this case is considered in the present paper. The relations between variables in both coordinate systems are given by the Galilean translation:

$$
t=\bar{t}, x=\bar{x}-t u_{s q}+\text { constant, } y=\bar{y}, u=\bar{u}-u_{s q}, v=\bar{v}, p=\bar{p}, T=\bar{T}
$$

In the local coordinate system, the equations (5) - (9) and the boundary conditions at the inlet and outlet (see Figure 1) remain the same as given for the first coordinate system.

Velocity-slip and temperature-jump boundary conditions are imposed on the walls of the channel and the square. The transition between steady and unsteady final state occurs at Knudsen numbers lower than 0.02. Therefore, we impose first order velocity-slip and temperature-jump BCs as follows:

$$
\begin{aligned}
& v_{s}-v_{w}=\left.A_{\sigma} \operatorname{Kn}_{\text {local }} \frac{\partial v}{\partial n}\right|_{s}, \\
& T_{s}-T_{w}=\left.\zeta_{T} \operatorname{Kn}_{\text {local }} \frac{\partial T}{\partial n}\right|_{s},
\end{aligned}
$$

where $v_{s}$ is velocity of the gas at the solid wall surface, $v_{w}$ is velocity of the wall, $\mathrm{Kn}_{\text {local }}=\mathrm{Kn} / \rho_{\text {local }}$ is the local Knudsen number, $\rho_{\text {local }}$ is the local density, $\left.\frac{\partial v}{\partial n}\right|_{s}$ is the derivative of velocity normal to the wall surface, $T_{s}$ is temperature of the gas at the wall surface, $T_{w}$ is temperature of the wall and $\left.\frac{\partial T}{\partial n}\right|_{s}$ is the derivative of temperature normal to the wall surface. We have used the velocity-slip and temperature-jump coefficients $A_{\sigma}=1.1466$ and $\zeta_{T}=2.1904$, calculated respectively in [16], [17], from the BGK equation by using variational method (for details see [18]).

\section{Computational considerations}

In our considerations, we are interested mainly in the final flow state established after passing the transient period of flow evolution. Two final 
regimes that occur in the flow are distinguished and the transition between them is analyzed:

Steady state regime: The flow regime after transient period of calculation is steady and macroscopic variable fields do not change in the course of time. Unsteady state regime: The flow regime after transient period of calculation is unsteady and macroscopic variable fields varies periodically in time (a non-periodical behavior is also possible but not observed in this investigation).

A transition (neutral) curve delineated in the space of parameters $M$ and Kn separates both steady and unsteady state regimes.

The final states of both regimes are investigated at Mach numbers from 0.1 to 0.6 for $\mathrm{B}=3,5,10$ and 20 . Results from an earlier stage of this study for the case $\mathrm{B}=3$ at Mach numbers $\mathrm{M}=0.1,0.2,0.3$ and 0.4 were presented in [19]. Here, a series of numerical calculations with different Knudsen number are performed for each considered Mach number. The simulation starts from a uniform state of the gas with temperature $T_{w}$, pressure $p_{\infty}$ and velocity equal to the channel walls. In SIMPLE-TS within each time step the iteration process is stopped, when the maximum residuals of the equations are less than $10^{-9}$. The final state is considered as unsteady when the latter criteria are not fulfilled in the course of time and non-symmetrical variations along $y$ axis are developed in the macroscopic variable fields. The non-symmetrical oscillations in the investigated cases are time dependent and lead to corresponding variations of the drag coefficient. We consider that the unsteady flow reaches a final state when the drag coefficient exhibits a regular periodic time evolution behavior in any cross-section behind the square, see Figure 2. The drag coefficient $C_{D}$ is defined as:

$$
C_{D}=\frac{F_{D}^{*}}{\frac{1}{2} \rho_{\infty}^{*} u_{s q}^{* 2} A^{*}},
$$

where $F_{D}^{*}$ is dimensional drag force, $u_{s q}^{*}$ is dimensional square velocity, and $A^{*}$ is dimensional cross section area of the square normal to the free stream flow. 


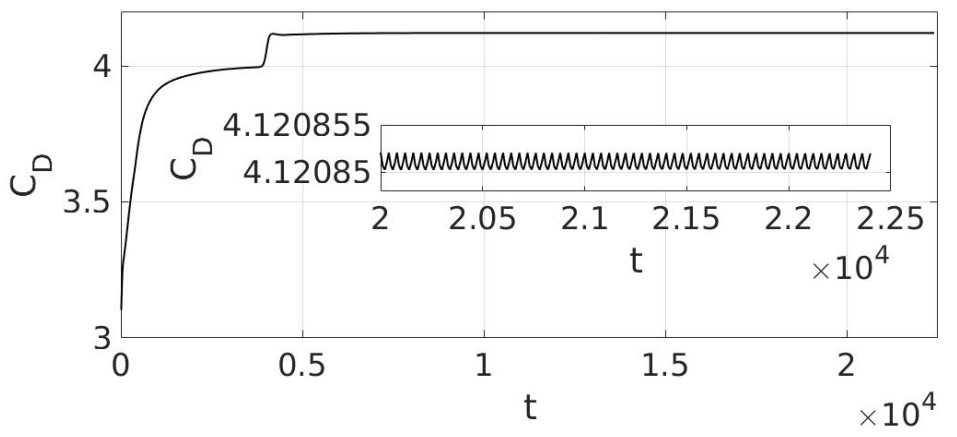

Figure 2: Time evolution of drag coefficient for $\mathrm{B}=3, \mathrm{M}=0.6, \mathrm{Kn}=0.008$.

An important requirement for accurate calculations is to realize a flow regime when the influence of inflow and outflow boundaries becomes negligible. It is worth noting, that for the continuum approach the implementation of inflow and outflow boundary condition with no fixed pressure keeping mass conservation in computational domain is a task, yet not achieved completely [20]. Thus, in our considerations the pressure at the inflow boundary is fixed. The influence of this boundary condition can be neglected, when the disturbances arising around the moving square do not reach the channel inflow area, see Figure 3.

The system of equations (5) - (9) is solved by using the algorithm

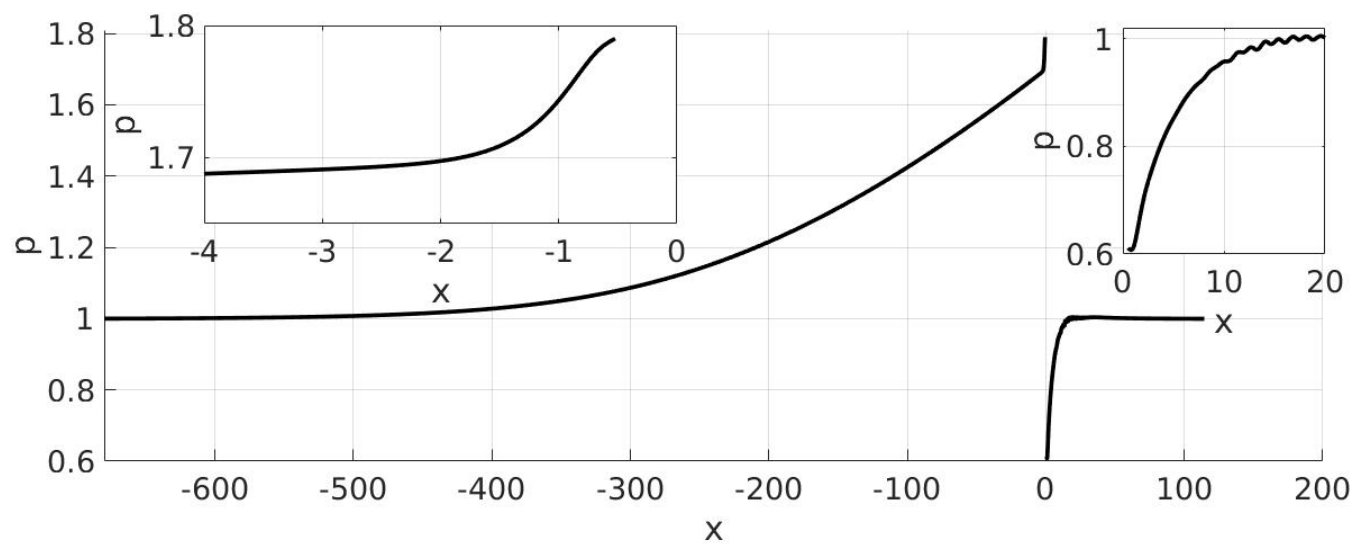

Figure 3: Pressure along the channel central line $(y=0)$ for $\mathrm{B}=3, \mathrm{M}=0.6$ and $\mathrm{Kn}=0.008$.

SIMPLE-TS [7]. Two variants of algorithm SIMPLE-TS were used. First one implies a Total Variation Diminishing (TVD) second order scheme, with SU- 
PERBEE limiter [21] that approximates convective terms. The TVD scheme was applied as explicit scheme (Forward Euler) with regard to time. The second one, upwind first order scheme, approximates convective terms as an implicit scheme (Backward Euler) with regard to time. A second order central difference implicit scheme (Backward Euler) approximates the diffusion terms. Subsonic cases were calculated using SIMPLE-TS with second order TVD scheme for approximation of convective terms. A GPU (Graphical Processor Unit) version of algorithm SIMPLE-TS [12] with Initial Guess Extrapolation (IGE) [13] was used to speed-up the calculations.

The SIMPLE-TS algorithm uses a staggered-mesh system. Two meshes were used to ensure sufficient accuracy of results and reasonable computational time. For cases $\mathrm{B}=3$ and Mach number $\mathrm{M} \geq 0.4$ and $\mathrm{B}=5$ and $\mathrm{M} \geq 0.55$, spatial steps along $\mathrm{x}$-axis $\Delta x_{\min }=0.05$ for $x \in[-10 ; 20]$ (around the square) and $\Delta x_{\max }=0.4$ for $x \leq-30$ and $x \geq 40$ were used while step along y-axis is constant $\Delta y=\Delta x_{\min }=0.05$. For smooth transition between $\Delta x_{\min }$ and $\Delta x_{\max }$ a linear polynomial relation was used. The uniform Cartesian mesh around the square, where gradients could be significant, is finer, while in zones with small gradients is coarser, see Figure 3. The staggered-mesh for all other considered cases was defined analogically with spatial steps: $\Delta x_{\min }=0.1, \Delta x_{\max }=0.75$ and $\Delta y=\Delta x_{\min }=0.1$. The time step is sufficiently small to ensure convergence of numerical algorithm SIMPLE-TS and so that it is in a range from 0.01 to 0.04 for subsonic considered cases.

In the present paper, the particle DSMC method was used for validation of the continual approach in the early transition regime. The Stefanov's Simplified Bernoulli trials (SBT) (see for details [22]) collision scheme was applied in the Transient Adaptive Sub-cells (TAS) [23] in order to improve the DSMC spatial accuracy. To be consistent with the continual approach, DSMC uses hard-sphere monoatomic gas collision model.

\section{Results}

We present mainly results for subsonic and transonic regimes because the calculations of a supersonic case required significant computational times because for transonic and supersonic regimes a flow transition occurs at very small Knudsen numbers between 0.001 and 0.0001. Additional complications raised for the continuum solver with second order TVD approximations of convective terms when non-physical oscillations appeared (especially at lower 
Knudsen numbers) in the numerical solution. This forced us to use first order upwind scheme requiring a very fine grid. As well-known the DSMC computational requirements also increase for lower Knudsen numbers. Nevertheless, the position of the shock wave in front of the square for $\mathrm{B}=3$ turned out to be a very sensitive parameter requiring a detailed computational analysis. To illustrate the later case, mesh and particles number convergence of SIMPLE-TS and DSMC as shown in Figure 4 for $\mathrm{Kn}=0.02$. It should be noted also that even the SIMPLE-TS was applied on fine grid the numerical solution was not sufficiently accurate in space to determine more sharply the position of the transition curve. To improve accuracy of the transition curve position, a new second order scheme and adaptive mesh was needed to determine place of the shock wave with reasonable computational resources. This circumstance explains why we restricted our detailed investigation up to $\mathrm{M}=0.6$.

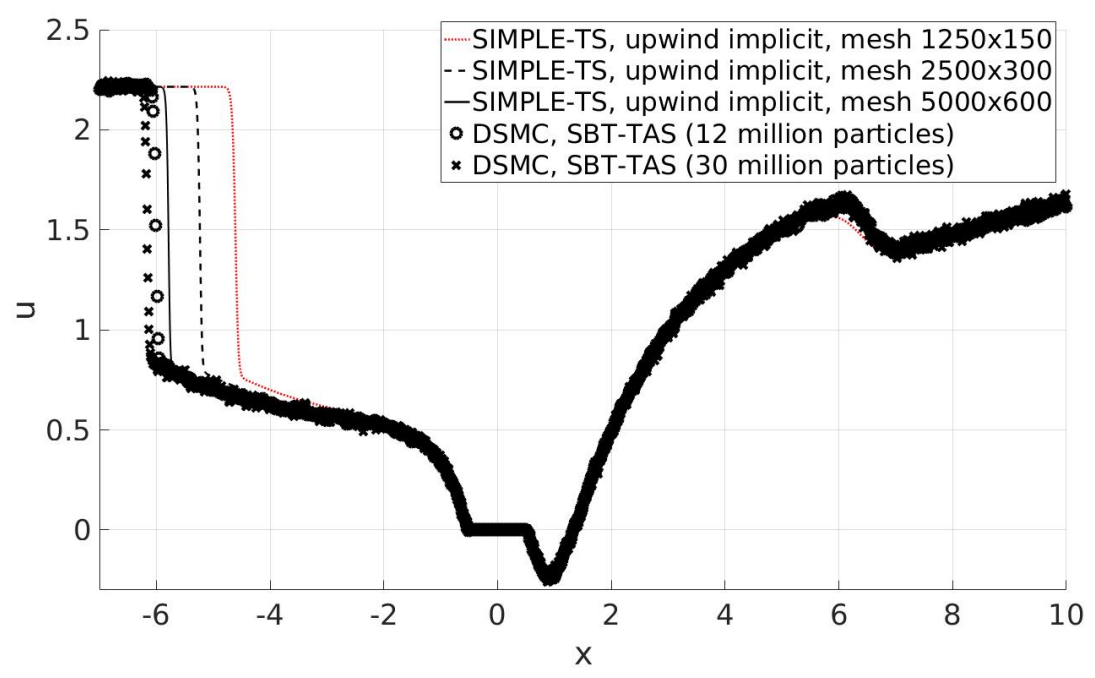

Figure 4: Horizontal nondimensional velocity along the channel central line $(y=0)$, comparison between SIMPLE-TS (continuum approach) and DSMC (molecular approach) with Transient Adaptive Subcell techniques TAS for $\mathrm{M}=2.43, \mathrm{Kn}=0.02$.

The comparisons between DSMC and Navier-Stokes-Fourier continuum model [6], [7], [24], show as expected a better agreement for smaller Knudsen numbers when the gas flow is close to continuum regime. Here we present a comparison between DSMC and SIMPLE-TS for $\mathrm{B}=3, \mathrm{M}=0.6$ and $\mathrm{Kn}=0.05$. The channel length for this case is $L_{c h}=300$ while the inlet area length is $L_{a}=200$. The DSMC calculation with 7.5 million particles in computational 
domain, Figure 5 and Figure 6, shows an excellent agreement with the calculated by using SIMPLE-TS macroscopic fields of $p, u, v, T$ and profiles of $p$ and $u$.

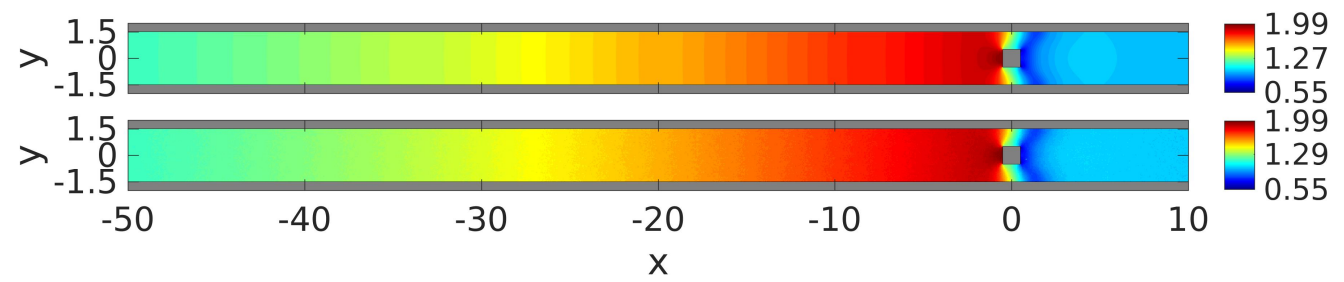

(a) $p$ obtained by SIMPLE-TS (upper part) and DSMC (lower part)

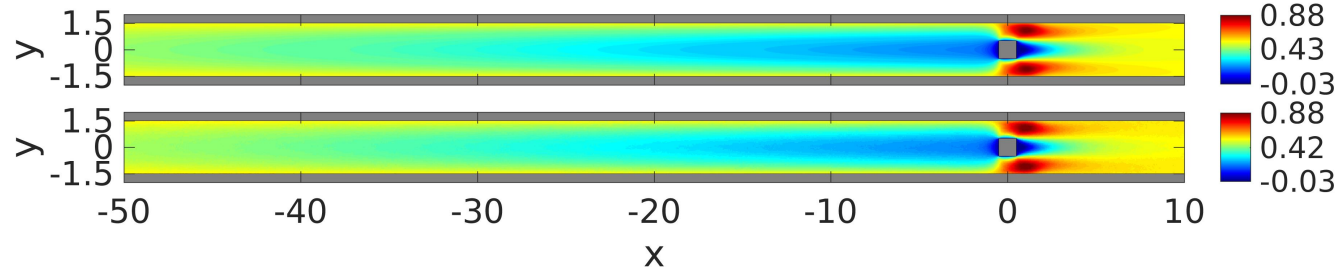

(b) $u$ obtained by SIMPLE-TS (upper part) and DSMC (lower part)

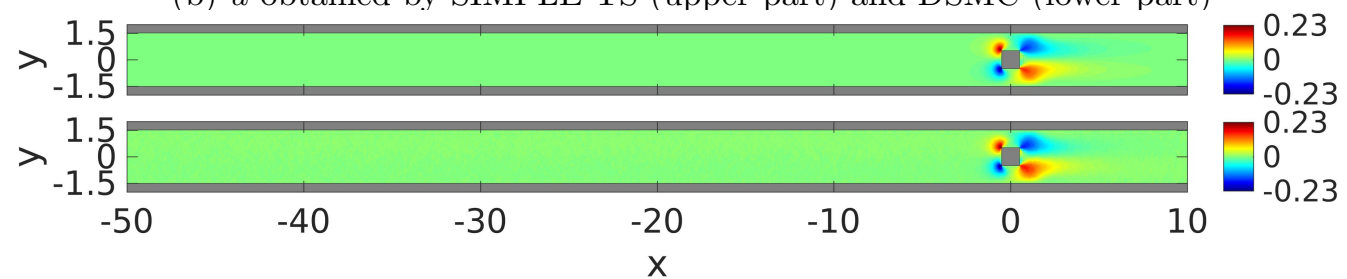

(c) $v$ obtained by SIMPLE-TS (upper part) and DSMC (lower part)

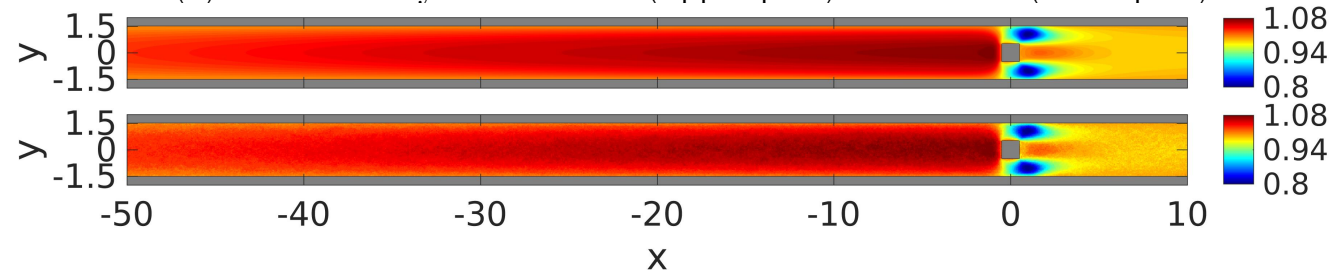

(d) $T$ obtained by SIMPLE-TS (upper part) and DSMC (lower part)

Figure 5: Comparison fields obtained by SIMPLE-TS and DSMC for $\mathrm{B}=3, \mathrm{M}=0.6$ and $\mathrm{Kn}=0.05$ for: (a) $p$, (b) $u$, (c) $v$ and (d) $T$. 


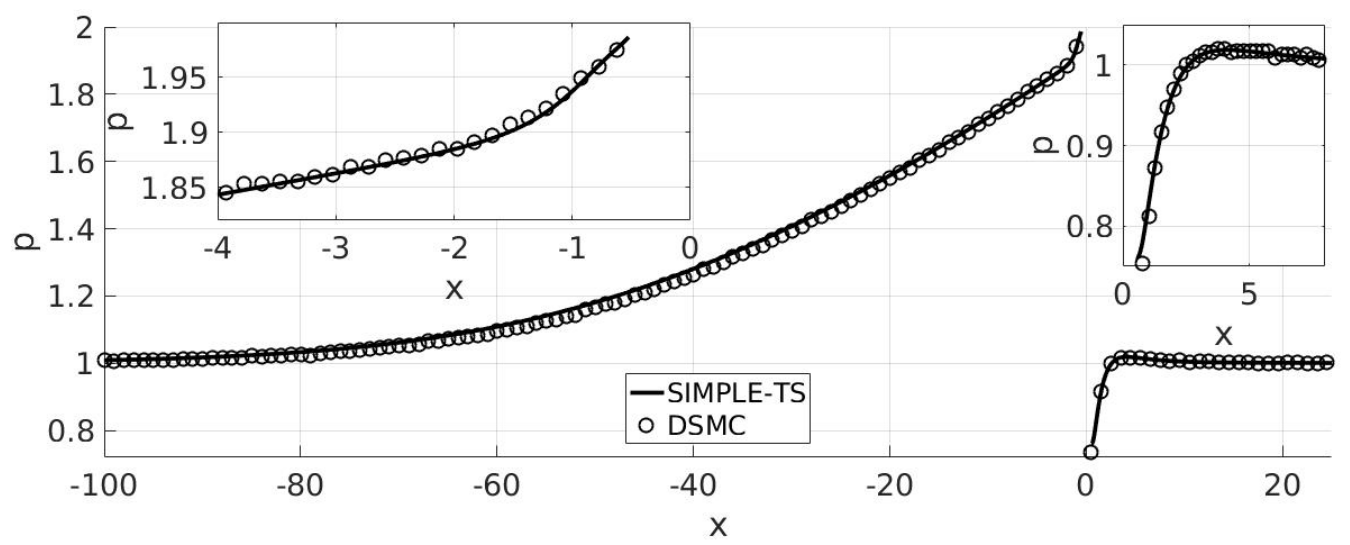

(a) Pressure

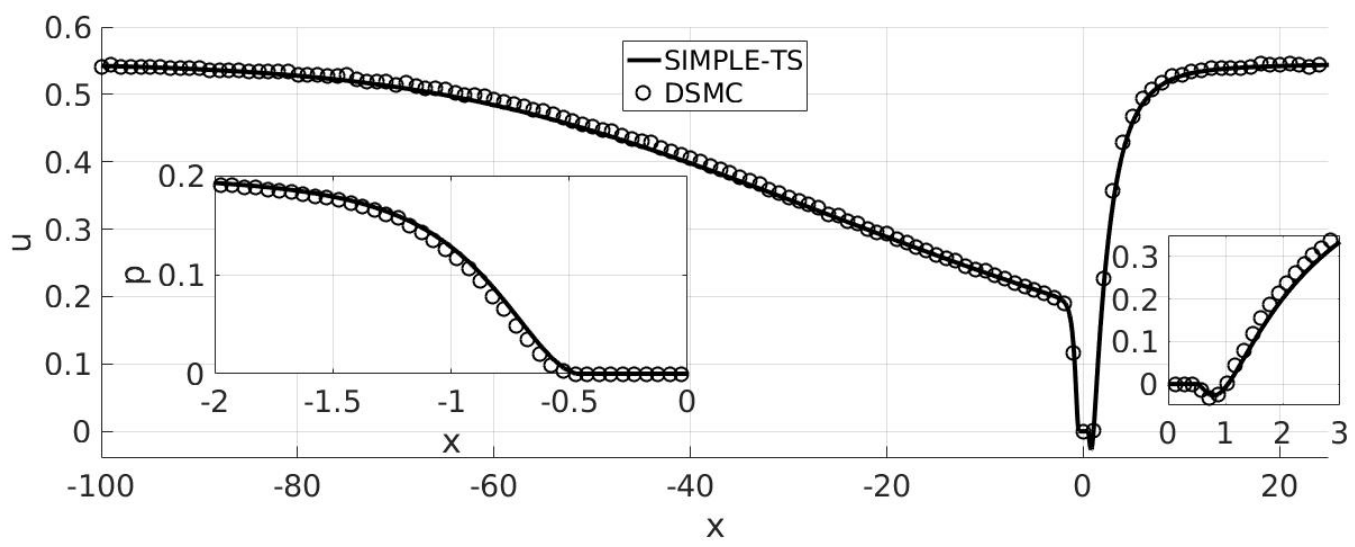

(b) Horizontal component of velocity

Figure 6: Comparison of profiles obtained by SIMPLE-TS (solid line) and DSMC (circles) for $\mathrm{B}=3, \mathrm{M}=0.6$ and $\mathrm{Kn}=0.05$ along the channel central line $(y=0)$ for: (a) pressure and (b) horizontal component of velocity. 
The blockage ratio $\mathrm{B}$ is bounded by two limits. One limit is $\mathrm{B}=1$ that corresponds to movement of a piston in a channel. The fluid does not pass around the square and the length of disturbances in front of the square (correspondingly, drag coefficient) increases unlimited. The other case is when B tends to infinity. This situation corresponds to a free stream flow past a moving square cylinder in unconfined domain. For the unconfined flow we have used appropriate boundary conditions at the bottom and top boundaries of computational domain replacing the walls with free stream conditions as follows: $p=1$, the other independent variables $u, v$ and $T$ were extrapolate linearly from the two closest non-ghost cells, or first-order extrapolation. The largest blockage ratio $\mathrm{B}=20$ considered in this paper is sufficient to neglect the boundary effects and replace the wall boundaries with the described above free stream boundary conditions. Figure 7 shows a comparison of pressure profiles along $\mathrm{x}$-axis between an unconfined flow past a square cylinder and the flow past a square confined in a microchannel for $\mathrm{B}=20$. The length of disturbances in front of the square differs significantly. We consider that disturbance length in front of the square $\left(L_{d}\right)$ is a distance from the front side of the square to the place where nondimensional pressure drop down to 1.001. length $L_{d}=74$ for the unconfined flow is significantly smaller then $L_{d}=6138$ for the flow past square in a microchannel. The difference between drag coefficients varies in wide range between $10-20$ percents for the considered range of Kn numbers, see Figure 8a. The results show that even for $\mathrm{B}=20$ the flow "feels" the walls and the boundary conditions have a significant impact on the macroscopic quantities, especially, on the length of disturbed area in front of the square cylinder.

Figure 8 shows the drag coefficient as a function of Knudsen number for $M=0.2,0.4$ and 0.6 and $B=3,5,10$ and 20. The Reynolds number is defined by the relation

$$
\mathrm{Re}=\frac{16}{5 \pi} \sqrt{\frac{\gamma \pi}{2}} \frac{\mathrm{M}}{\mathrm{Kn}} \approx \sqrt{\frac{\gamma \pi}{2}} \frac{\mathrm{M}}{\mathrm{Kn}},
$$

which is valid for compressible viscous model at small Knudsen numbers (see [25]).

The local Mach number has been expressed using nondimensional variables as follows:

$$
\mathrm{M}_{i, j}^{\text {local }}=\sqrt{\frac{2\left(u_{i, j}^{2}+v_{i, j}^{2}\right)}{\gamma T_{i, j}}}, \text { for } i=1,2 \ldots N x \text {, and } j=1,2 \ldots N y,
$$




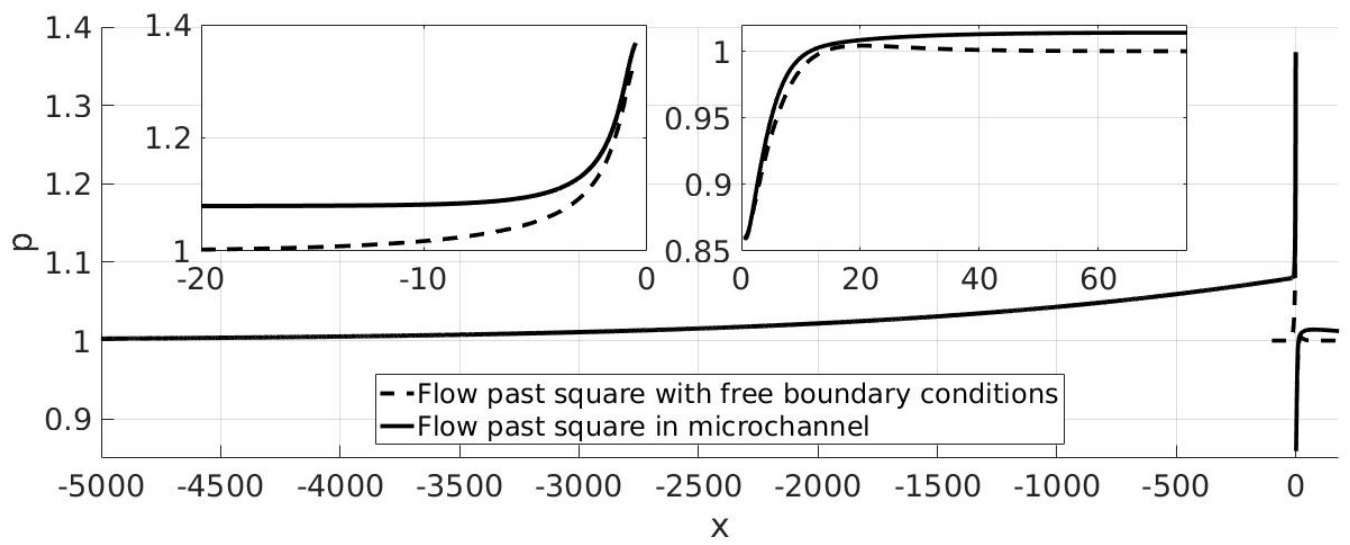

Figure 7: Pressure along the channel central line $(y=0)$ for $\mathrm{B}=20, \mathrm{M}=0.6$ and $\mathrm{Kn}=0.02$. Note: The square size (equal to 1) compared to examined disturbance length (5000) is small and hardly visible. The pressure profile in front of the square is given from channel inlet to $x=-0.5$ while the pressure profile after the square is from $x=0.5$ to the channel outlet.

where $N x$ and $N y$ are number of mesh nodes along x-axis and y-axis respectively.

The blockage ratio affects significantly flow properties such as drag coefficient, pressure at stagnation point of the square and the transition point between flow regimes. The dependence of the drag coefficient on the Knudsen number for different Mach numbers (in the same sub-figure) and different blockage ratios (in different sub-figures) is given in Figure 8. The range of values of each graphic is chosen large enough to capture the transition point between the flow regimes, where the drag coefficient curve undergoes a jump or a sudden change of its behavior when the flow transforms from one mode to another. According to eq. (14) the drag coefficient could be presented versus Reynolds number. However, as one can see Re, Mach and Kn are coupled and this does not allow to illustrate the effect of rarefaction on the transition point. In the vicinity of the transition point the flow behind the square in the steady state forms two symmetric vortices. With decrease of $\mathrm{Kn}$ at the same Mach the vortices become longer, respectively, more unstable and the flow looses the symmetry in this zone. The critical regime starts with a separation of the longer vortex followed by the separation of the second one. This mechanism is repeated periodically and the Karman vortex street is formed. In the zone, where the flow is stable the drag coefficient behavior is typical and it increases when the Knudsen number increases. On 
the other hand, the smaller blockage ration leads to a larger drag coefficient $\left(C_{D}\right)$. With respect to the Knudsen number, the lower blockage ratio moves the critical transition point left, to lower Knudsen numbers, while the higher Mach number moves it right, to higher Kn values. Thus, the drag coefficient $C_{D}$ in the critical transition point for $\mathrm{B}=20$ is around 1.65 to 1.72 for the considered Mach numbers. For $\mathrm{B}=10$ the transition point moves to values of $C_{D}$ around 1.8-1.9, for $\mathrm{B}=5$ to $2.44-2.55$ and for $\mathrm{B}=3$ the drag coefficient increase is up to 4.05-4.35 for considered Mach numbers. The drag coefficient $C_{D}$ at the transition point increases when Mach number increases. An exception case is $C_{D}$ for $\mathrm{B}=3$ and $\mathrm{M}=0.6$ because the gas flow is transonic (see Figure 10), while in all other cases considered in Figure 8 the flow is subsonic. For $\mathrm{B}=3$ and $\mathrm{M}=0.6$ the drag coefficient $C_{D}$ have 3 parts: $C_{D}^{A}$, $C_{D}^{B}$ and $C_{D}^{C}$. Part $C_{D}^{A}$ corresponds to a steady state regime at larger $\mathrm{Kn}$, where $C_{D}$ monotonically decreases when Kn decreases. For intermediate Kn, in the zone of the transition point from steady to unsteady-state regime the neutral curve changes its slope and starts increasing when Kn decreases (part $\left.C_{D}^{B}\right)$. The drag in this part has small-amplitude but the energy conditions are changing sweepingly to jump on the third branch with $C_{D}^{C}$, see Figure 9a. Its slope changes once again for part $C_{D}^{C}$ and $C_{D}$ decreases when Kn decreases in part $C_{D}^{A}$. $C_{D}$ oscillations in $C_{D}^{B}$ increase significantly according to oscillations in part $C_{D}^{B}$ for small change of Knudsen number, see Figure $9 \mathrm{~b}$. 


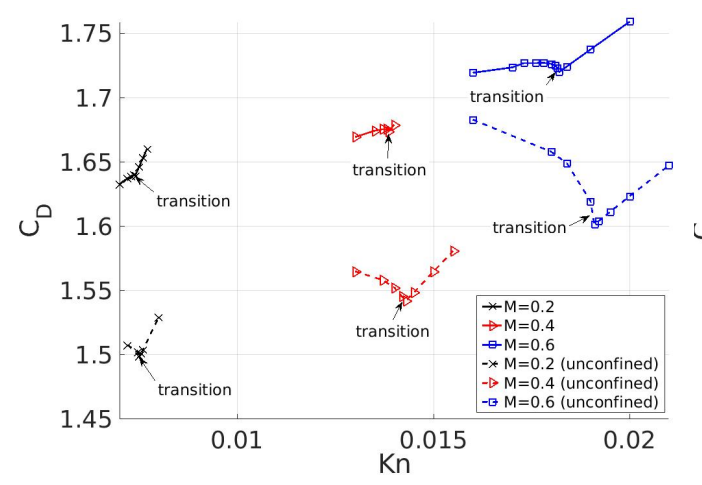

(a) $\mathrm{B}=20$ and unconfined flow past a square

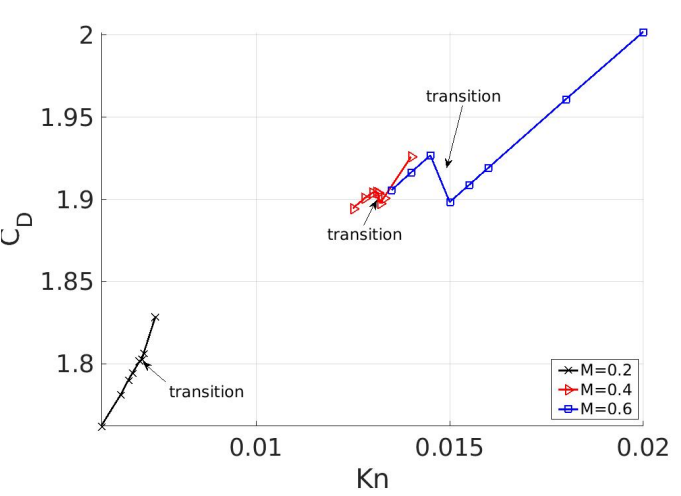

(b) $\mathrm{B}=10$

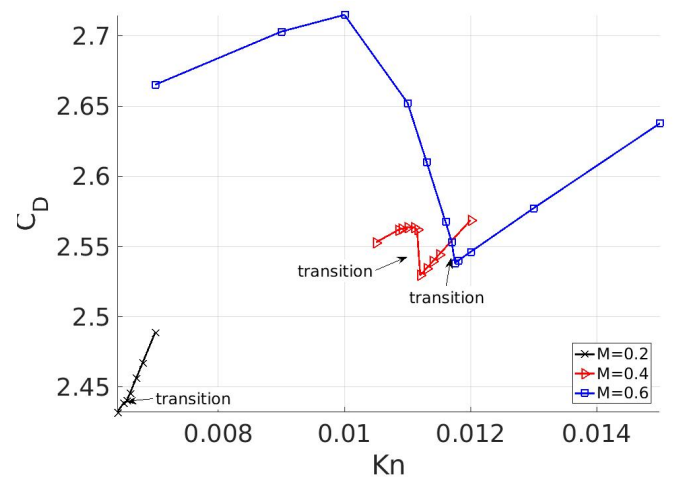

(c) $\mathrm{B}=5$

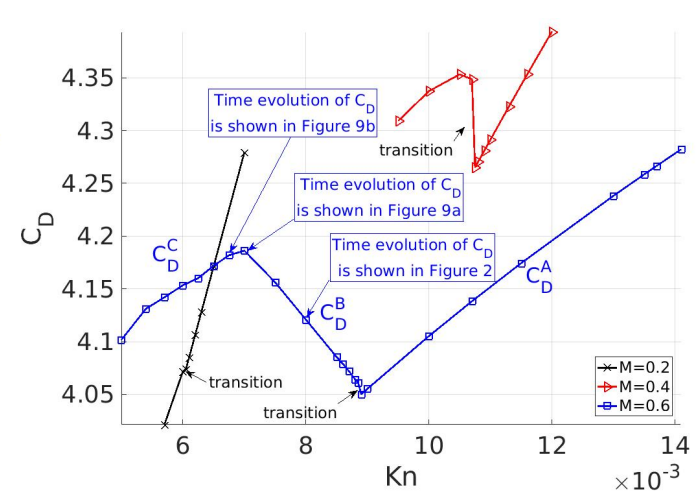

(d) $\mathrm{B}=3$

Figure 8: Drag coefficient $\left(C_{D}\right)$ as a function of Knudsen number $(\mathrm{Kn})$ for the cases of $\mathrm{M}=0.2,0.4$ and 0.6 and unconfined flow and blockage ratio $\mathrm{B}=20 \mathrm{a}), \mathrm{B}=10 \mathrm{~b}), \mathrm{B}=5 \mathrm{c}$ ), and $\mathrm{B}=3 \mathrm{~d})$.

Note: the drag coefficient curve is separated into two parts with respect to the transition point: the left part represents the drag coefficient in unsteady regimes while the right part - in steady-state regimes. 


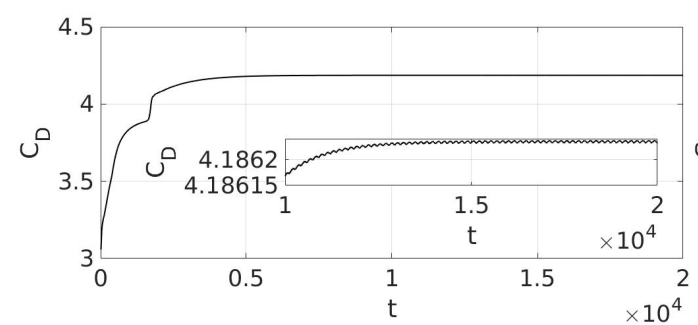

(a) $\mathrm{Kn}=0.007$

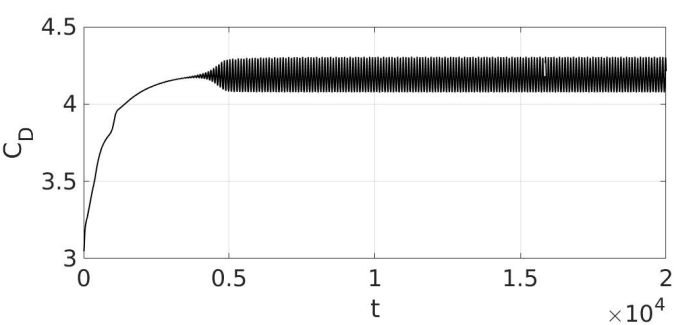

(b) $\mathrm{Kn}=0.00675$

Figure 9: Time evolution of drag coefficient $\left(C_{D}\right)$ as function of Knudsen number $(\mathrm{Kn})$ for Mach numbers $\mathrm{M}=0.6$ and blockage ratio $\mathrm{B}=3$ and $\mathrm{Kn}=0.007$ a) and $\mathrm{Kn}=0.00675 \mathrm{~b}$ ).

Lower blockage ratio decreases the cross section around the square, increases the velocity around the square and shift back the maximum velocity regions observed on both sides of the square, see Figure 10.

Figures 10, 11 and 12 illustrate local Mach number, pressure and temperature flow fields for unconfined case (a) and different blockage ratios (b), (c), (d), and (e). The Knudsen varies in the range between 0.01 and 0.02 . The figures demonstrate the developed Karman vortex street past the square for this range of Knudsen numbers. The influence of the channel walls is also clearly seen when compared to the unconfined case (a). Figure 13 shows pressure variation at stagnation point for the closest to the transition point steady state regime. The pressure at stagnation point increases when B decreases as expected. The gas flow in a microchannel at low Mach number $(\mathrm{M}=0.1)$ leads to long propagation of small disturbances in front of the channel because the flow regime is close to incompressible limit. The precise calculations require extremely long microchannel and a lot of computational resources. A shorter channel did not contain the full length of disturbances but it determines the pressure at stagnation point within negligible differences compared to a much longer channel and one cannot see any difference in the position of the transition point between steady and unsteady state regime. On other hand, when the Mach number goes up the disturbance length decreases but the impact on the pressure at the stagnation point is bigger, see Figure 13. The inlet length cutoff for $M=0.4$ shows that a pressure difference at the stagnation point of $12 \%$ resulting in moving the trasnition point with respect to Knudsen number by about $20 \%$. This short analysis demonstrates the importance of the pressure calculation at stagnation point within required accuracy. One can find a proportionality of the distances 


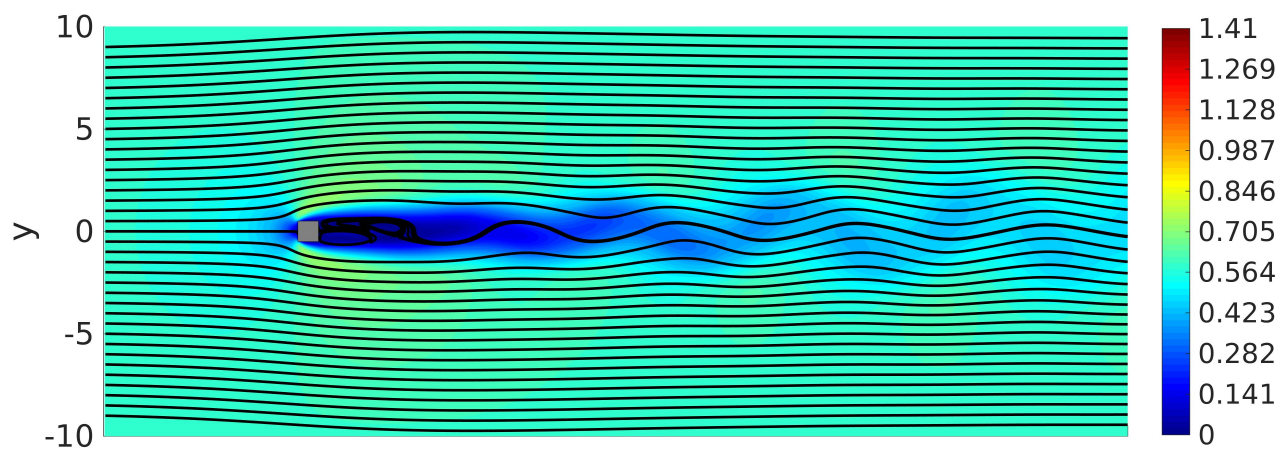

(a) Unconfined flow past a square and $\mathrm{Kn}=0.0190$

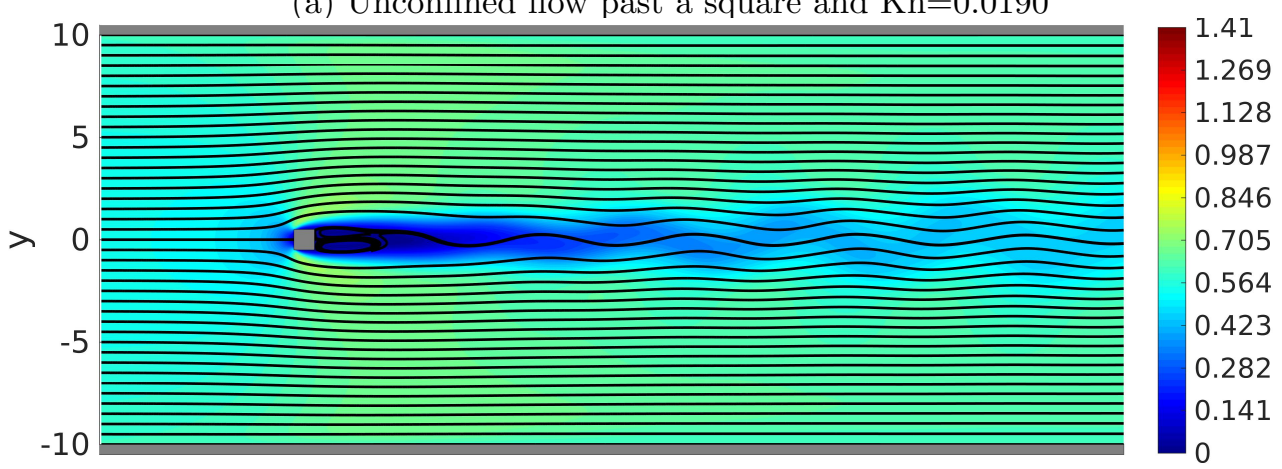

(b) $\mathrm{B}=20$ and $\mathrm{Kn}=0.01815$

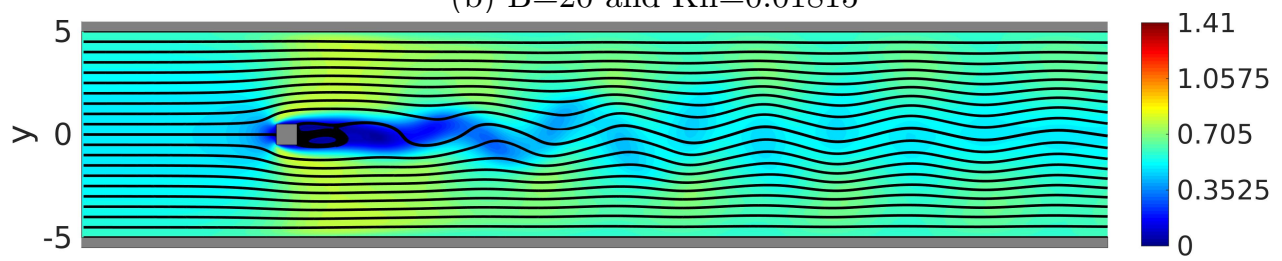

(c) $\mathrm{B}=10$ and $\mathrm{Kn}=0.0145$

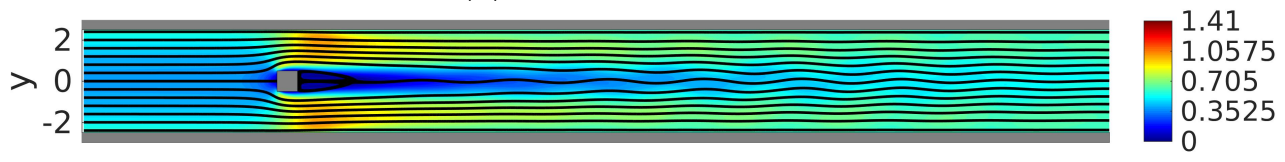

(d) $\mathrm{B}=5$ and $\mathrm{Kn}=0.0114$

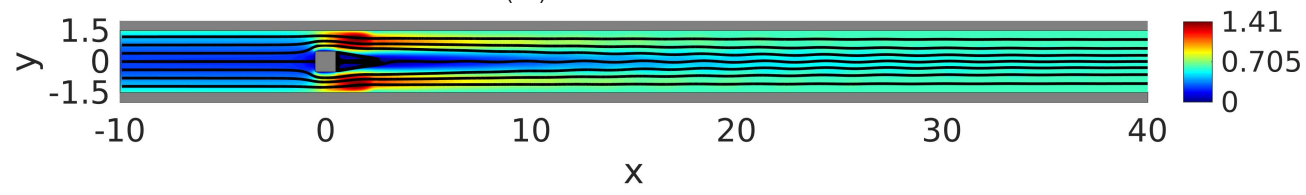

(e) $\mathrm{B}=3$ and $\mathrm{Kn}=0.00885$

Figure 10: Unsteady state regime local Mach number fields and streamlines obtained closest to the transition curve for Mach number 0.6 and unconfined flow past a square and $\mathrm{Kn}=0.0190 \mathrm{a}$ ), $\mathrm{B}=20$ and $\mathrm{Kn}=0.01815 \mathrm{~b}$ ), $\mathrm{B}=10$ and $\mathrm{Kn}=0.0145 \mathrm{c}$ ), $\mathrm{B}=5$ and $\mathrm{Kn}=0.0114$ $\mathrm{d})$, and $\mathrm{B}=3$ and $\mathrm{Kn}=0.00885 \mathrm{e}$ ). 


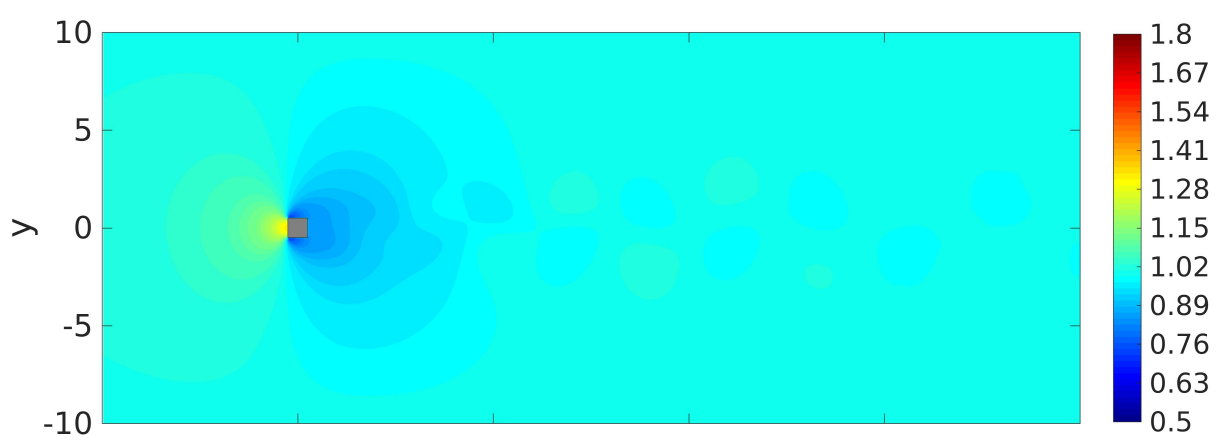

(a) Unconfined flow past a square and $\mathrm{Kn}=0.0190$

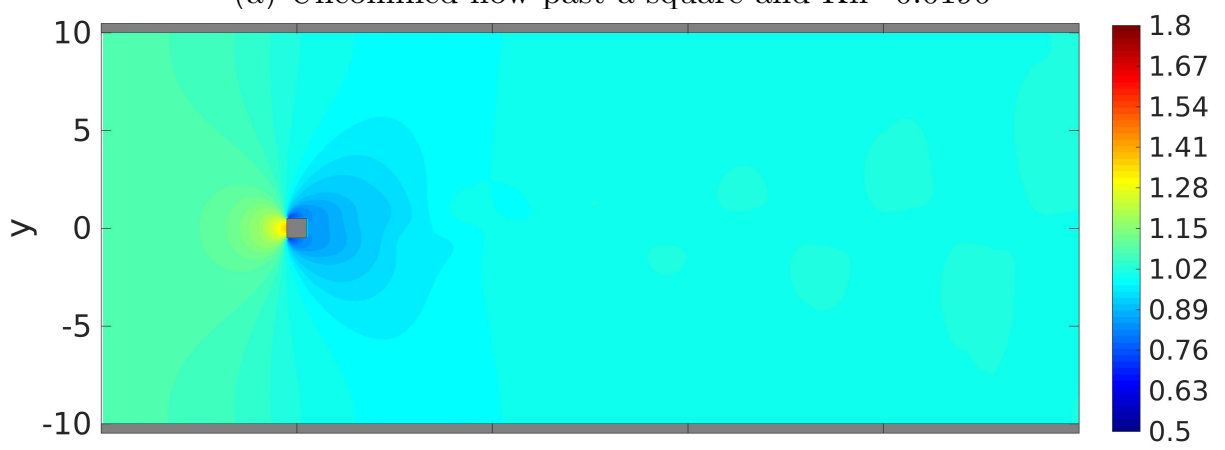

(b) $\mathrm{B}=20$ and $\mathrm{Kn}=0.01815$

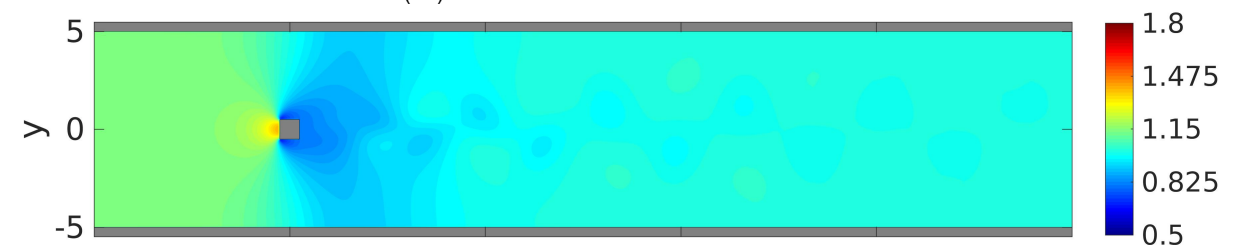

(c) $\mathrm{B}=10$ and $\mathrm{Kn}=0.0145$

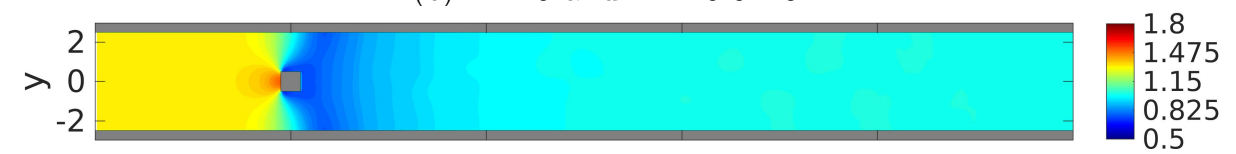

(d) $\mathrm{B}=5$ and $\mathrm{Kn}=0.0114$

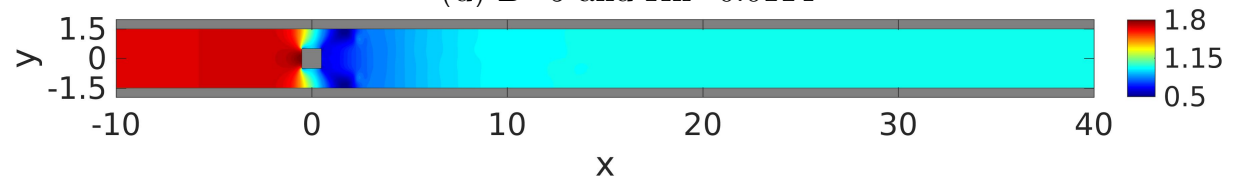

(e) $\mathrm{B}=3$ and $\mathrm{Kn}=0.00885$

Figure 11: Unsteady state regime pressure field obtained closest to the transition curve for Mach number 0.6 and unconfined flow past a square and $K n=0.0190$ a), $B=20$ and $\mathrm{Kn}=0.01815 \mathrm{~b}$ ), $\mathrm{B}=10$ and $\mathrm{Kn}=0.0145 \mathrm{c}$ ), $\mathrm{B}=5$ and $\mathrm{Kn}=0.0114 \mathrm{~d}$ ), and $\mathrm{B}=3$ and $\mathrm{Kn}=0.00885 \mathrm{e})$. 


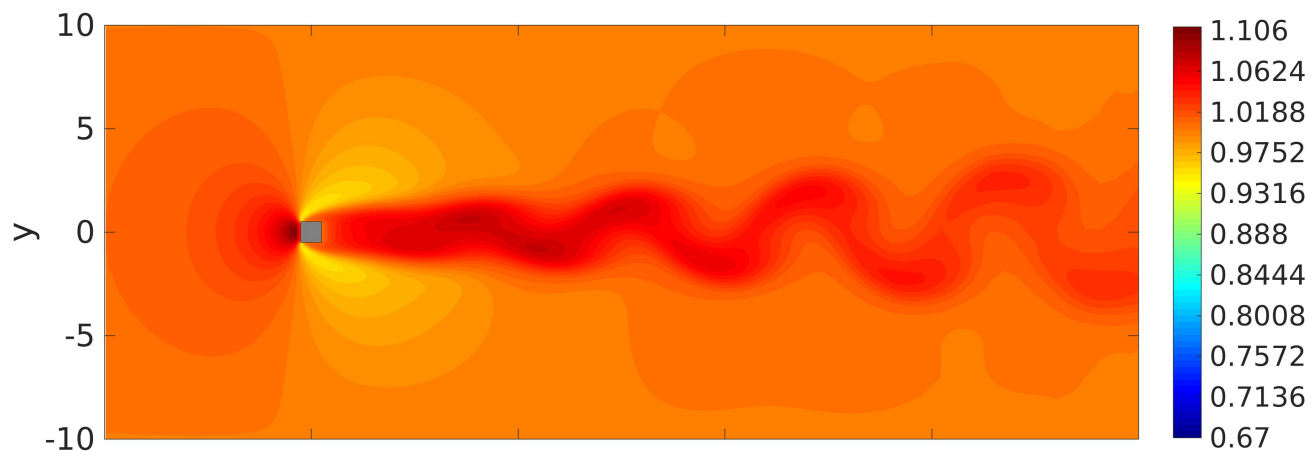

(a) Unconfined flow past a square and $\mathrm{Kn}=0.0190$

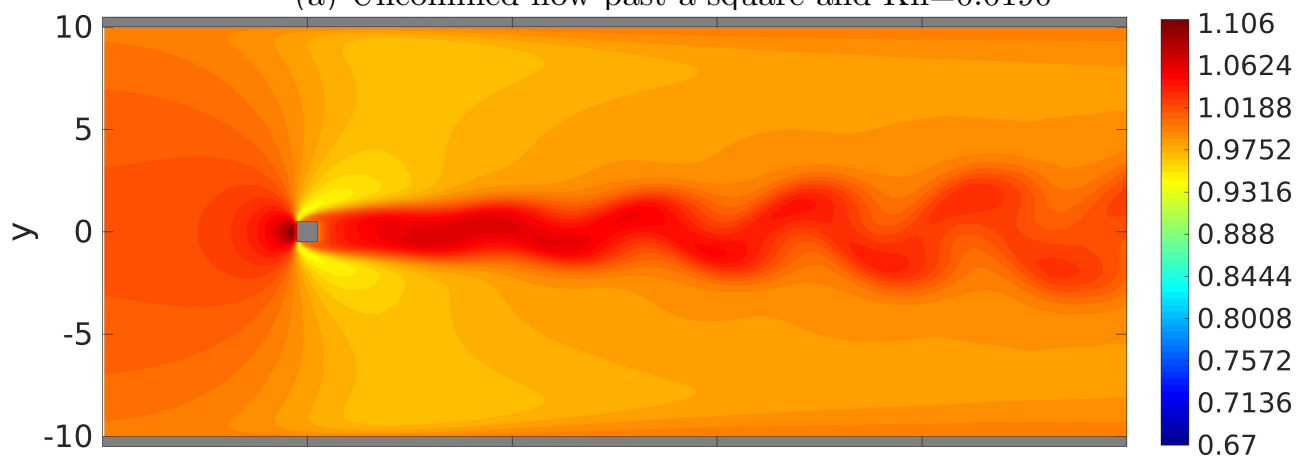

(b) $\mathrm{B}=20$ and $\mathrm{Kn}=0.01815$

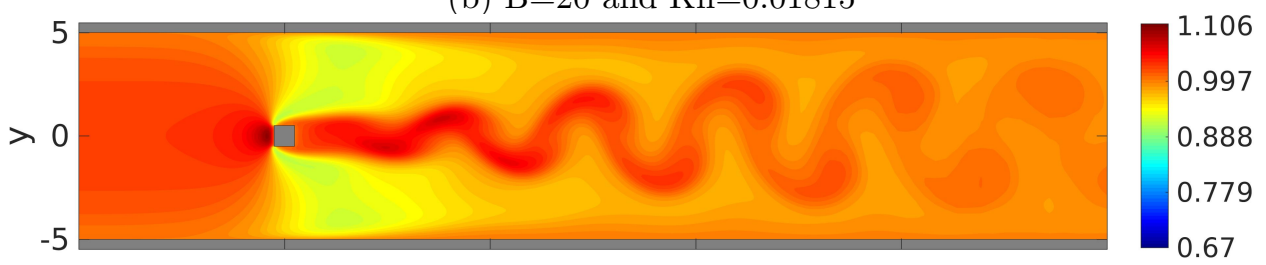

(c) $\mathrm{B}=10$ and $\mathrm{Kn}=0.0145$

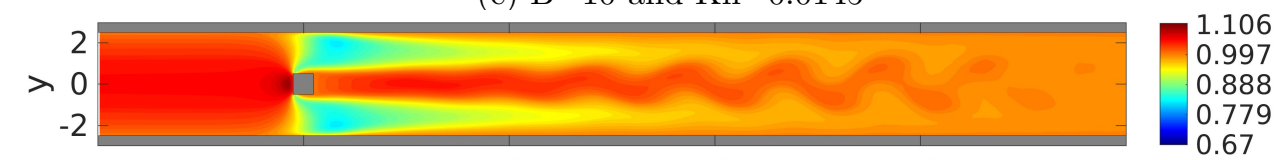

(d) $\mathrm{B}=5$ and $\mathrm{Kn}=0.0114$

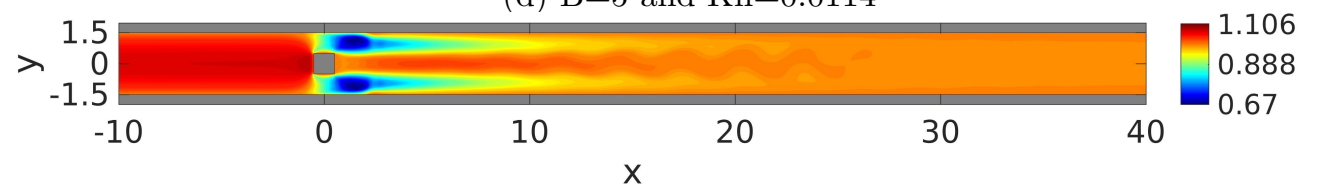

(e) $\mathrm{B}=3$ and $\mathrm{Kn}=0.00885$

Figure 12: Unsteady state regime temperature field obtained closest to the transition curve for Mach number 0.6 and unconfined flow past a square and $\mathrm{Kn}=0.0190$ a), $\mathrm{B}=20$ and $\mathrm{Kn}=0.01815 \mathrm{~b}$ ), $\mathrm{B}=10$ and $\mathrm{Kn}=0.0145 \mathrm{c}$ ), $\mathrm{B}=5$ and $\mathrm{Kn}=0.0114 \mathrm{~d}$ ), and $\mathrm{B}=3$ and $\mathrm{Kn}=0.00885 \mathrm{e})$. 
between the pressure curves and the distances between corresponding transition curves on Figure 14a.

The transition curve shape is similar for all considered B (Figure 14).

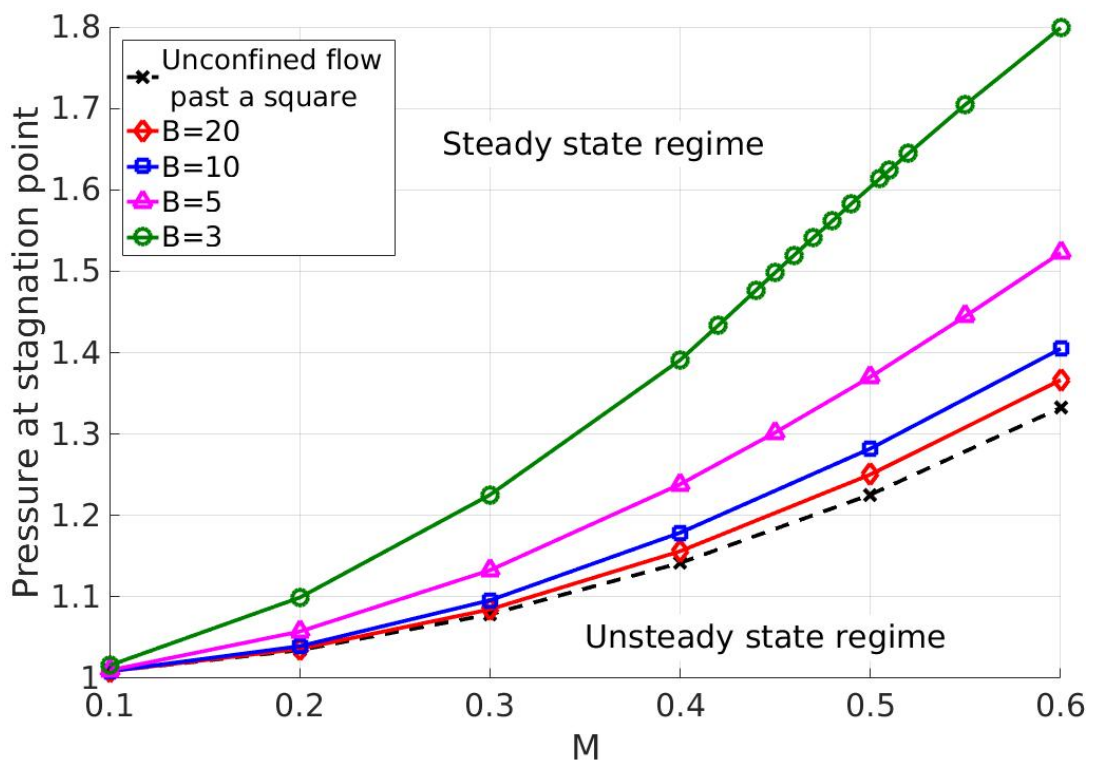

Figure 13: Pressure at stagnation point vs Mach number.

When the blockage ratio is smaller the channel walls are closer to the square walls and stabilizes the gas flow (see Figure 10, Figure 11 and Figure 12). This effect shifts the corresopnding transition curve to lower Knudsen numbers. On the other hand, the smaller B leads to an increase of velocity near the square. This effect shifts the transition curve to lower Mach numbers. 


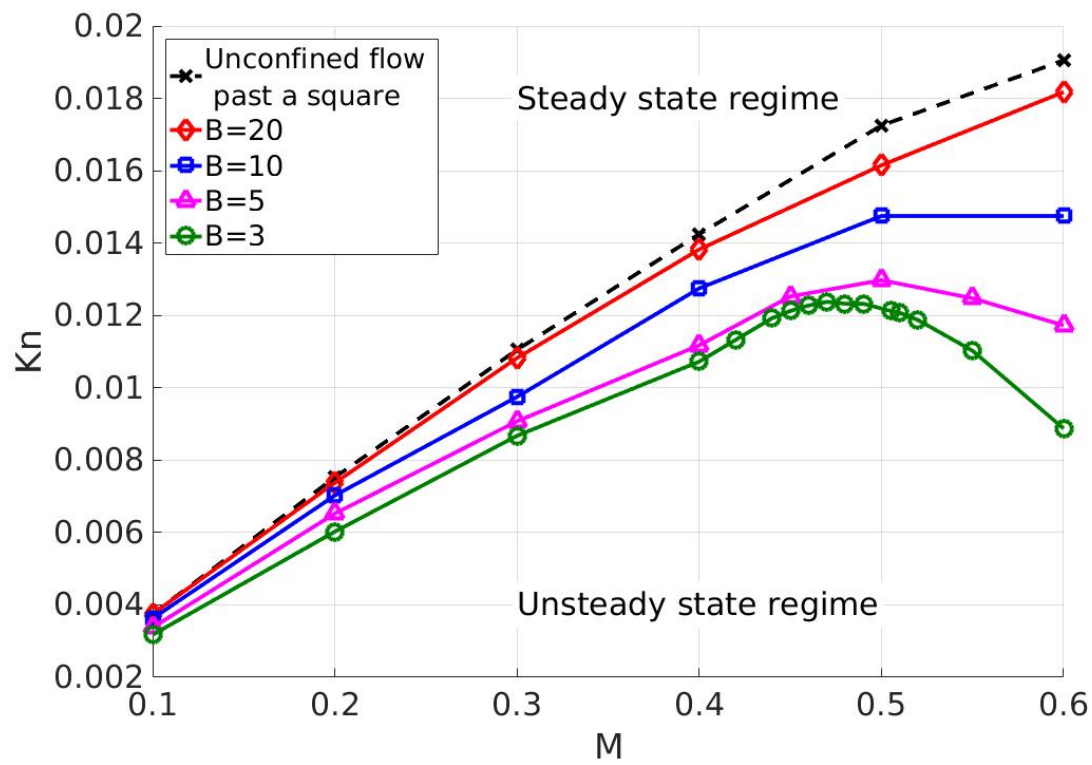

(a) The transition (neutral) curve obtained for unconfined flow past a square and flow past square confined in a microchannnel for $\mathrm{B}=20,10$, 5 and 3 and subsonic regimes

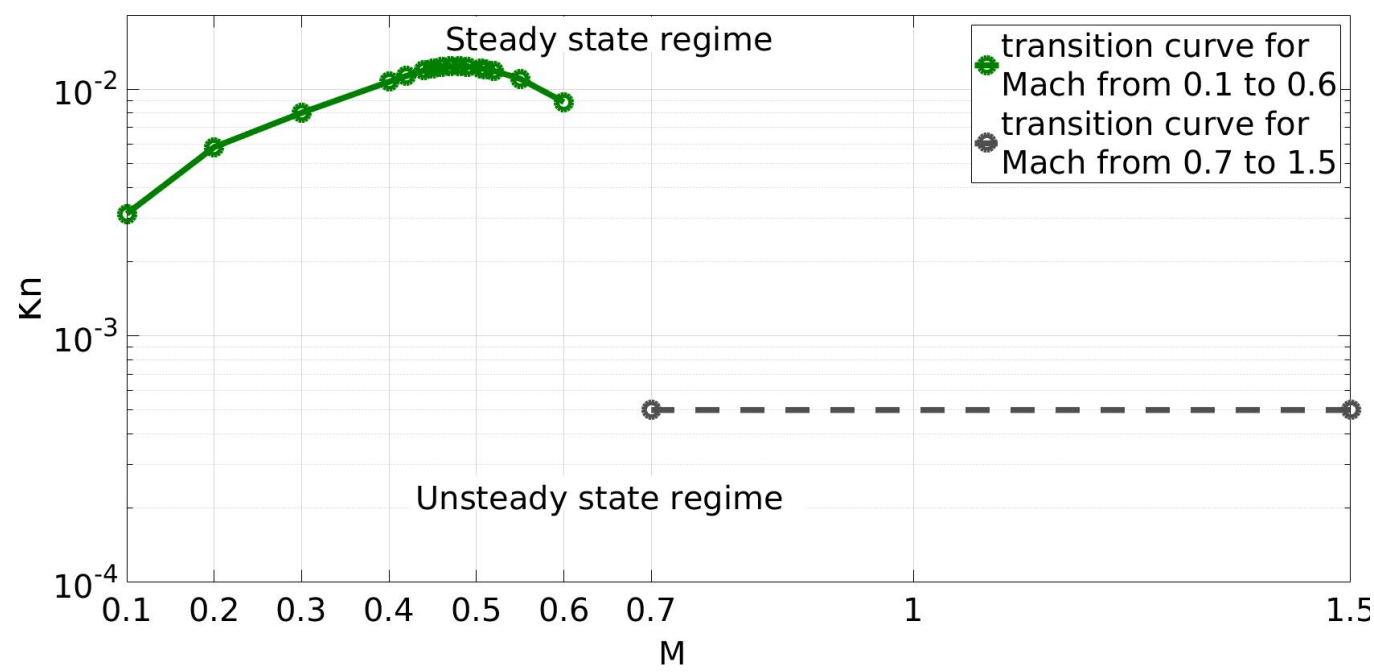

(b) The transition (neutral) curve obtained for flow past square confined in a microchannnel $(\mathrm{B}=3)$ for subsonic, transonic and supersonic regimes

Figure 14: The transition (neutral) curve as a function of Knudsen and Mach numbers. 


\section{Conclusions}

We analyzed the influence of blockage ratio on the transition between steady and unsteady final state regimes of gas flow past a square cylinder in a microchannel. The results obtained by continuum model (SIMPLETS solver) were compared to the DSMC data for the case $B=3, M=0.6$ and $\mathrm{Kn}=0.05$ showing an excellent agreement with the molecular model. The comparisons between unconfined and confined square in a microchannel illuminated the fact that even for large blockage ratio as $\mathrm{B}=20$ the influence of channel walls on the flow around the square is significant. Presented are the transition curves for blockage ratios $\mathrm{B}=3, \mathrm{~B}=5, \mathrm{~B}=10$, and $\mathrm{B}=20$. The analysis showed that the transition curves for considered blockage ratios possess similar shape and the decrease of B leads to shifting of the transition curve to lower Knudsen and Mach numbers.

\section{Acknowledgements}

We would like to acknowledge the financial support provided by the Bulgarian NSF under Grant DN-02/7-2016. This research has received funding from the European Union Horizon 2020 research and innovation programme under the Marie Sklodowska-Curie MIGRATE grant agreement No. 643095.

\section{References}

[1] S. Colin, Rarefaction and compressibility effects on steady and transient gas flows in microchannels, Microfluidics and Nanofluidics 1 (2005) 268279. doi:10.1007/s10404-004-0002-y.

URL http://dx.doi.org/10.1007/s10404-004-0002-y

[2] M. Nabavi, Steady and unsteady flow analysis in microdiffusers and micropumps: a critical review, Microfluidics and Nanofluidics 7 (2009) 599-619. doi:10.1007/s10404-009-0474-x.

URL http://dx.doi .org/10.1007/s10404-009-0474-x

[3] C. Cercignani, Rarefied Gas Dynamics. From Basic Concept to Actual Calculations, University press, Cambridge, 2000.

[4] Y. Sone, Molecular Gas Dynamics: Theory, Techniques, and Applications, Birkhäser, Boston, 2007.

[5] G. A. Bird, Molecular, Gas Dynamics and the Direct Simulation of Gas Flows, Clarendon Press, Oxford, 1994. 
[6] S. Stefanov, V. Roussinov, C. Cercignani, Rayleigh-Bénard flow of a rarefied gas and its attractors. I. Convection regime, Physics of Fluids 14 (7) (2002) 2255-2269. doi:10.1063/1.1483837.

URL http://link.aip.org/link/?PHF/14/2255/1

[7] K. S. Shterev, S. K. Stefanov, Pressure based finite volume method for calculation of compressible viscous gas flows, Journal of Computational Physics 229 (2) (2010) 461-480. doi:10.1016/j.jcp.2009.09.042.

URL http://dx.doi.org/10.1016/j.jcp.2009.09.042

[8] V. C. Patel, W. Rodi, G. Scheuerer, Turbulence models for near-wall and low Reynolds number flows-a review, AIAA Journal 23 (9) (1985) 1308-1319. doi:10.2514/3.9086.

URL http://dx.doi.org/10.2514/3.9086

[9] J. Johansen, J. Sørensen, Prediction of laminar/turbulent transition in airfoil flows, Journal of aircraft 36 (4) (1999) 731-734. doi:10.2514/2.2501. URL http://dx.doi.org/10.2514/2.2501

[10] C. N. A. Sohankar, L. Davidson, Numerical Simulation of Unsteady Flow Around a Square Two-Dimensional Cylinder, 1995, pp. 517-520. URL http://www.tfd.chalmers.se/ lada/postscript_files/sydney_paper_ahmad.pdf

[11] C.-H. Cheng, F.-L. Liao, DSMC Analysis of Rarefied Gas Flow Over a Rectangular Cylinder at All Knudsen Numbers, Journal of Fluids Engineering 122 (2000) 720 - 729. doi:10.1115/1.1315301.

URL http://dx.doi.org/10.1115/1.1315301

[12] K. S. Shterev, E. I. Atanassov, S. K. Stefanov, GPU Calculations of Unsteady Viscous Compressible and Heat Conductive Gas Flow at Supersonic Speed, Springer Berlin Heidelberg, Berlin, Heidelberg, 2014, pp. 549-556. doi:10.1007/978-3-662-43880-0_63.

URL http://dx.doi.org/10.1007/978-3-662-43880-0_63

[13] K. S. Shterev, Iterative process acceleration of calculation of unsteady, viscous, compressible, and heat-conductive gas flows, International Journal for Numerical Methods in Fluids 77 (2) (2015) 108-122. doi:10.1002/fld.3979.

URL http://dx.doi.org/10.1002/fld.3979 
[14] S. Stefanov, V. Roussinov, C. Cercignani, Rayleigh-Bénard flow of a rarefied gas and its attractors. II. Chaotic and periodic convective regimes, Physics of Fluids 14 (7) (2002) 2270-2288. doi:10.1063/1.1483839.

URL http://link.aip.org/link/?PHF/14/2270/1

[15] S. Stefanov, V. Roussinov, C. Cercignani, Rayleigh-Bénard flow of a rarefied gas and its attractors. III. Three-dimensional computer simulations, Physics of Fluids 19 (12) (2007) 124101. doi:10.1063/1.2815729. URL http://link.aip.org/link/?PHF/19/124101/1

[16] S. Albertoni, C. Cercignani, L. Gotusso, Numerical evaluation of the slip coefficient, Physics of Fluids 6 (7) (1963) 993-996. doi:http://dx.doi.org/10.1063/1.1706857.

URL http://scitation.aip.org/content/aip/journal/pof1/6/7/10.1063/1.1706857

[17] P. Bassanini, C. Cercignani, C. Pagani, Comparison of kinetic theory analyses of linearized heat transfer between parallel plates, International Journal of Heat and Mass Transfer 10 (4) (1967) $447-460$. doi:http://dx.doi.org/10.1016/0017-9310(67)90165-2.

URL http://www.sciencedirect.com/science/article/pii/0017931067901652

[18] C. Cercignani, The Boltzmann Equation and its Applications, Springer, New York, 1988.

[19] K. Shterev, S. Stefanov, Determination of Zone of Flow Instability in a Gas Flow Past a Square Particle in a Narrow Microchannel, in: M. Dulea, A. Karaivanova, A. Oulas, I. Liabotis, D. Stojiljkovic, O. Prnjat (Eds.), High-Performance Computing Infrastructure for South East Europe's Research Communities, Vol. 2 of Modeling and Optimization in Science and Technologies, Springer International Publishing, 2014, pp. 43-50. doi:10.1007/978-3-319-01520-0_5.

URL http://dx.doi.org/10.1007/978-3-319-01520-0_5

[20] M. GadelHak, MEMS: Introduction and Fundamentals, CRC Press, 2005.

[21] P.L.Roe, Some contributions to the modelling of discontinuous flows, Lectures in Applied Mathematics 22 (1985) 163-193. 
[22] S. K. Stefanov, On DSMC Calculations of Rarefied Gas Flows with Small Number of Particles in Cells, SIAM Journal on Scientific Computing 33 (2) (2011) 677-702. arXiv:http://dx.doi.org/10.1137/090751864, doi:10.1137/090751864.

URL http://dx.doi.org/10.1137/090751864

[23] G. A. Bird, Visual DSMC Program for Two-Dimensional and Axially Symmetric Flows, The DS2V Program Users Guide, Ver. 2.1, G.A.B. Consulting Pty Ltd, Sydney, Australia, 2003.

[24] K. Shterev, S. Stefanov, Strouhal number analysis for a Karman vortex gas flow past a square in a microchannel at low mach number, AIP Conference Proceedings 1629 (1) (2014) 319-326. doi:http://dx.doi.org/10.1063/1.4902288. URL http://scitation.aip.org/content/aip/proceeding/aipcp/10.1063/1.4902288

[25] S. K. Stefanov, R. W. Barber, M. Ota, D. R. Emerson, Comparison between Navier-Stokes and DSMC Calculations for Low Reynolds Number Slip Flow Past a Confined Microsphere, AIP Conference Proceedings 762 (1) (2005) 701-706. doi:10.1063/1.1941617.

URL http://link.aip.org/link/?APC/762/701/1 